\title{
Identification of reference genes in blood before and after entering the plateau for SYBR green RT-qPCR studies
}

\author{
Jun Xiao $^{1}{ }^{\text {, Xiaowei Li }}{ }^{1}{ }$ Juan Liu $^{1}{ }^{\text {, Xiu Fan }}{ }^{1}$, Huifen Lei ${ }^{1}$, Cuiying Li ${ }^{\text {Corresp. }}{ }^{1}$ \\ 1 Department of Blood Transfusion, General Hospital of Air Force, PLA, Beijing, China \\ Corresponding Author: Cuiying Li \\ Email address: licuiying2013@qq.com
}

Background: Tibetans have lived at high altitudes for thousands of years, and they have unique physiological traits that enable them to tolerate this hypoxic environment. However, the genetic basis of these traits is still unknown. As a sensitive and highly efficient technique, RT-qPCR is widely used in gene expression analyses to provide insight into the molecular mechanisms underlying environmental changes. However, the quantitative analysis of gene expression in blood is limited by a shortage of stable reference genes for the normalization of mRNA levels. Thus, systematic approaches were used to identify potential reference genes.

Results: The expression levels of eight candidate human reference genes (GAPDH, ACTB, $18 S$ RNA, $\beta 2$ $M G, P P I A, R P L 13 A, T B P$ and SDHA) were assessed in blood from hypoxic environments. The expression stability of these selected reference genes was evaluated using the geNorm, NormFinder and BestKeeper programs. Interestingly, RPL13A was identified as the ideal reference gene for normalizing target gene expression in human blood before and after exposure to high-altitude conditions.

Conclusion: These results indicate that different reference genes should be selected for the normalization of gene expression in blood from different environmental settings. 


\section{Identification of reference genes in blood before and after entering the plateau}

3 Jun Xiao

4 Affiliation: Department of Blood Transfusion, General Hospital of Air Force, PLA, 30 Fucheng

5 Road, Beijing 100142, P R China

6 E-mail:ammsxj@126.com

7 Xiaowei Li

8 Affiliation: Department of Blood Transfusion, General Hospital of Air Force, PLA, 30 Fucheng

9 Road, Beijing 100142, P R China

10 E-mail:lixiaowei0825@126.com

11 Juan Liu

12 Affiliation: Department of Blood Transfusion, General Hospital of Air Force, PLA, 30 Fucheng

13 Road, Beijing 100142, P R China

14 E-mail:juanliu903@163.com

15 Xiu Fan

16 Affiliation: Department of Blood Transfusion, General Hospital of Air Force, PLA, 30 Fucheng

17 Road, Beijing 100142, P R China

18 E-mail: fanxiu0304@yahoo.com

19 Huifen Lei

Affiliation: Department of Blood Transfusion, General Hospital of Air Force, PLA, 30 Fucheng

E-mail:value_0302@126.com

Cuiying Li*(corresponding author)

*Authors to whom correspondence should be addressed

Affiliation: Department of Blood Transfusion, General Hospital of Air Force, PLA, 30 Fucheng Road, Beijing 100142, P R China

E-Mails: licuiying2013@qq.com

29

Tel.: +86-10-6692-8461

Fax. : $+86-10-6698-7754$ 
30 Abstract

31 Background: Tibetans have lived at high altitudes for thousands of years, and they have unique physiological traits that enable them to tolerate this hypoxic environment. However, the genetic basis of these traits is still unknown. As a sensitive and highly efficient technique, RT-qPCR is widely used in gene expression analyses to provide insight into the molecular mechanisms underlying environmental changes. However, the quantitative analysis of gene expression in blood is limited by a shortage of stable reference genes for the normalization of mRNA levels. Thus, systematic approaches were used to identify potential reference genes.

Results: The expression levels of eight candidate human reference genes (GAPDH, ACTB, $18 S$ RNA, $\beta 2-M G, P P I A, R P L 13 A, T B P$ and SDHA) were assessed in blood from hypoxic environments. The expression stability of these selected reference genes was evaluated using the geNorm, NormFinder and BestKeeper programs. Interestingly, RPL13A was identified as the ideal reference gene for normalizing target gene expression in human blood before and after 43 exposure to high-altitude conditions.

44 Conclusion: These results indicate that different reference genes should be selected for the 45 normalization of gene expression in blood from different environmental settings. 


\section{Introduction}

Hypoxia is a major biological feature of high-altitude regions (Beall 2000). In hypoxic environments, transcription of various genes, such as endothelial PAS domain-containing protein 1 (EPAS1) and prolyl hydroxylase domain-containing protein 2 (PHD2), is initiated by hypoxiarelated pathways. An increasing number of studies show that the hypoxia-inducible factor (HIF) signaling pathway plays a vital role in the adaptation to hypoxia (Ji et al. 2012). The human EPAS1 gene encodes the alpha subunit of HIF-2 (HIF-2 $\alpha$ ), which acts as a key regulator of chronic hypoxia by regulating a large number of genes (Beall et al. 2010).

To examine the molecular mechanisms involved in these processes, quantitative gene expression analysis is indispensable. Quantitative real-time PCR (RT-qPCR) is a highly sensitive, precise and reproducible method for the detection of gene expression levels (Bustin 2002; Bustin \& Nolan 2004; Vandesompele et al. 2002). However, to produce optimal results from RT-qPCR analysis, minimum requirements must be met, including quality control of the mRNA and primers, PCR efficiency determination and selection of the appropriate reference genes (Nolan et al. 2006). The obtained gene expression profile varies based on the use of different housekeeping genes as internal references genes (Sellars et al. 2007). Therefore, proper reference gene selection guarantees the accuracy of the analysis data obtained from RT-qPCR (Vandesompele et al. 2002).

Researchers have always empirically determined reference genes, such as GAPDH and $\beta$-actin, during quantitative gene expression analyses. However, recent studies have shown that housekeeping gene (HKG) expression levels vary between cell types (Gentile et al. 2016; Ofinran et al. 2016; Wang et al. 2015) and experimental conditions (Tricarico et al. 2002; Zhang et al. 2005). Thus, a stable and suitable reference gene must be selected for the normalization of target gene expression.

In the present study, three algorithms (geNorm, NormFinder and BestKeeper) were utilized to analyze the stability of selected candidate reference genes [glyceraldehyde-3-phosphate dehydrogenase (GAPDH), $\beta$-actin (ACTB), $18 S$ RNA, $\beta 2$-microglobulin $(\beta 2-M G)$, peptidylprolyl isomerase A (PPIA), ribosomal protein L13 (RPL13A), TATA-Box binding protein (TBP) and succinate dehydrogenase complex, subunit $A$ (SDHA)] in human blood before and after exposure to high-altitude conditions using RT-qPCR with SYBR green.

\section{Materials and methods}

\section{Sample information}

Six healthy male Han Chinese volunteers $(21.3 \pm 1.3$ years old) who have in the plains (altitude $500 \mathrm{~m}$ ) for at least 20 years were enrolled. Blood samples were collected when they lived in the plains and 3 days after they moved onto the plateau (altitude 4,700 $\mathrm{m}$ ). They did not show any clinical signs of hypoxia at the time of the examination. This study was approved by the Institutional Review Board of the General Hospital of the Air Force, PLA (afgh-IRB-16-03). Each of the six volunteers provided written informed consent. 


\section{RNA samples and cDNA synthesis}

Mononuclear cells were isolated from $5 \mathrm{ml}$ of peripheral blood (before and after moving to the plateau, 3,700 m) by using lymphocyte separation medium (Solarbio, Beijing, China), as

previously described (Chen et al. 2016). Total RNA was extracted from $10^{7}$ mononuclear cells using TRIzol Reagent (Invitrogen) according to the manufacturer's protocol and then quantified using a UV-2550 spectrophotometer (Shimadzu). cDNA was synthetized from approximately 0.5 $\mu \mathrm{g}$ of total RNA using a ReverTra Ace ${ }^{\circledR} q \mathrm{qCR}$ RT kit with gDNA Remover (TOYOBO, Osaka, Japan).

\section{Candidate genes and primers for RT-qPCR}

Eight candidate human reference genes, GAPDH, ACTB, $18 S$ RNA, $\beta 2-M G$, PPIA, RPL13A, TBP and $S D H A$, were selected for evaluation based on the Minimum Information for Publication of Quantitative Real-Time PCR Experiments (MIQE) guidelines (Bustin et al. 2009) (Table 1). BLAST software was used to design the specific primers and to confirm the specificity of the primer sequences for the indicated gene. All primers, except for $18 S R N A$ and $\beta 2-M G$, spanned one intron to exclude the contamination of genomic DNA in total RNA.

\section{SYBR green real-time quantitative $R T-P C R$}

PCR was performed using a CFX-96 thermocycler PCR system (Bio-Rad). In each run, $1 \mu 1$ of synthetized cDNA was added to $19 \mu \mathrm{l}$ of reaction mixture containing $8 \mu \mathrm{l}$ of $\mathrm{H}_{2} \mathrm{O}, 10 \mu \mathrm{l}$ of THUNDERBIRD qPCR Mix (TOYOBO) and $0.5 \mu \mathrm{l}$ of forward and reverse primers $(10 \mu \mathrm{M})$. Each sample was measured in triplicate. PCR was conducted at $95^{\circ} \mathrm{C}$ for 3 min followed by 40 cycles of $95^{\circ} \mathrm{C}$ for $10 \mathrm{~s}, 58^{\circ} \mathrm{C}$ for $15 \mathrm{~s}$ and $72^{\circ} \mathrm{C}$ for $15 \mathrm{~s}$. The amplification was followed by melting curve analysis.

\section{Amplification efficiency and primer specificity of the reference genes}

The amplification efficiency (E) of the primers was tested using a standard curve RT-qPCR of a serially diluted $(1 / 10,1 / 100,1 / 1,000,1 / 10,000$, and 1/100,000) cDNA sample with the formula $\mathrm{E}$ $(\%)=\left(10^{-1 / \text { slope }}-1\right) \times 100$ (Ahn et al. 2008). The efficiency (E) and correlation coefficient $\left(R^{2}\right)$ of each candidate reference gene were calculated to determine amplification efficiency (Table 2). An amplification efficiency of $90-110 \%$ and an $\mathrm{R}^{2}$ of 0.99 were acceptable.

\section{Analysis of reference gene expression stability}

The geNorm (Vandesompele et al. 2002) program was used to measure gene expression stability (M), and this method differs from model-based approaches by comparing genes based on the similarity of their expression profiles. geNorm ranks the genes based on $\mathrm{M}$ values, where the gene with the most stable expression has the lowest value. NormFinder (Andersen et al. 2004) was used to find two genes with the least intra- and inter-group expression variation. A BestKeeper index was created using the geometric mean of the $\mathrm{Ct}$ values of each candidate gene. An estimation of the reference gene stability could be obtained by analyzing the calculated 
variation (standard deviation and coefficient variance) (Pfaffl et al. 2004).

Finally, RefFinder, a comprehensive web-based tool that integrates geNorm, NormFinder and BestKeeper, was applied to determine the most stable reference gene for the final ranking (Liu et al. 2015).

\section{Results}

\section{Determining the specificity and amplification efficiency of the primers}

The expression stability of eight candidate reference genes in subjects before and after migrating onto the plateau was analyzed using RT-qPCR. For each reference gene, primer specificity was demonstrated by a single peak in the melting curve analysis (Fig. 1). Amplification efficiencies were calculated as previously described (Ahn et al. 2008) and ranged from $95.6 \%$ to $114.7 \%$ for the eight reference genes. The correlation coefficient $\left(\mathrm{R}^{2}\right)$ of the standard curve for each gene was greater than 0.98 (Table 2).

\section{Expression levels of reference genes in the blood before and after migrating onto the plateau}

To examine the stability of eight HKGs before and after migrating onto the plateau, the expression levels were evaluated by RT-qPCR, and the Shapiro-Wilk test was used to evaluate the normality of the $\mathrm{Ct}$ values (Table 3). The $\mathrm{Ct}$ values ranged from 13.40 (ACTB) to $21.34(T B P)$ for the blood samples before ascending to the plateau (Table 3 and Fig. 2A) and 13.60 (RPL13A) to $21.78(T B P)$ for the samples taken after ascending to the plateau (Table 3 and Fig. 2B). ACTB and $R P L 13 A$ were more abundantly expressed than the other genes before and after migrating onto the plateau (Fig. 2).

\section{Candidate reference gene stability: geNorm}

Candidate reference gene stability was evaluated based on the $\mathrm{M}$ values of the genes using the geNorm algorithm (Vandesompele et al. 2002). The M values for GAPDH, ACTB, 18S RNA, $\beta 2$ $M G, P P I A, R P L 13 A, T B P$ and SDHA were lower than 1.5 in all samples. According to the analysis, $G A P D H$ and $A C T B$ were the most stable among all eight candidate genes on the plains (Fig. 3A), whereas $18 S R N A$ and RPL13A were the most stable genes on the plateau (Fig. 3B). Analysis of samples from both stages confirmed that GAPDH and RPL13A were the most stable genes (Fig. 3C).

Using the geNorm algorithm, the pairwise variation value $\left(\mathrm{V}_{\mathrm{n}} / \mathrm{V}_{\mathrm{n}+1}\right)$ was used to calculate the optimum number of reference genes for accurate normalization and to determine whether the addition of another reference gene $(n+1)$ for normalization was recommended. A cut-off threshold $\left(\mathrm{V}_{\mathrm{n}} / \mathrm{V}_{\mathrm{n}+1}=0.15\right)$ was used to determine the optimal number of reference genes required for normalization (Vandesompele et al. 2002). The greater the number of reference genes used for normalization, the more confidence there is in their gene expression level (Jaramillo et al. 2017). Two reference genes were sufficient for gene expression analysis of the blood in the plains (Fig. 3D) and plateau stages (Fig. 3E). When all samples were analyzed together, the $\mathrm{Vn} /$ $\mathrm{Vn}+1$ values ranged from 0.062 to 0.110 and were all lower than the threshold value of 0.15 
165

166

167

168

169

170

171

172

173

174

175

176

177

178

179

180

181

182

183

184

185

186

187

188

189

190

191

192

193

194

195

196

197

198

199

200

201

202

203

204

(Fig. 3F). Thus, only two HKGs are required for the normalization of target genes in expression analyses.

\section{Candidate reference gene stability: NormFinder}

The NormFinder algorithm ranks the HKGs according to the inter- and intra-group variations in expression (Ahn et al. 2008). The results indicated that GAPDH, PL13A, ACTB and PPIA in the plains group (Table 4) and PPIA, SDHA, ACTB and RPL13A in the plateau group (Table 4) were the most stable reference genes. PPIA, SDHA, TBP and RPL13A were the four most stable reference genes in both groups (Table 4).

\section{Candidate reference gene stability: BestKeeper}

The BestKeeper algorithm (Pfaffl et al. 2004) uses the coefficient variance (CV) and standard deviation (SD) of candidate gene expression to determine the optimal HKGs (Table 5). In the BestKeeper program, HKGs with lower SD and CV values are considered as optimal reference genes. In both stages, RPL13A expression had the lowest SD (0.15) and the lowest CV (1.10). Therefore, $R P L 13 A$ was proposed as the ideal HKG for the analysis of gene expression during the plains and plateau stages.

\section{Candidate reference gene stability: RefFinder}

Based on the geNorm, NormFinder and BestKeeper results, RefFinder (http://fulxie.0fees.us/) was used to calculate a comprehensive expression stability ranking. As shown in Table 6, GAPDH (plains) and PPIA (plateau) were the most stable HKGs before and after entering the plateau, respectively. Across both stages, PPIA and RPL13A were the most stable reference genes for the normalization of target gene expression levels.

\section{Discussion}

Understanding the mechanisms of high-altitude hypoxic adaptation is a major focus of highaltitude medical research. Using RT-qPCR to rapidly and accurately analyze gene expression is a common strategy for understanding the mechanisms of this process (Valasek \& Repa 2005). Since the expression levels of reference genes in endothelial cells (Bakhashab et al. 2014), epithelial cells (Liu et al. 2016) and cancer cells (Fjeldbo et al. 2016; Lima et al. 2016) can vary under hypoxic conditions, gene expression was analyzed in blood from subjects at various altitudes to determine which reference genes should be used under particular conditions. Most expression studies of blood under hypoxic conditions have used a single traditional reference gene, such as GAPDH, ACTB and I8S RNA (Polotsky et al. 2015; Srikanth et al. 2015), without evaluating the expression stability of these reference genes. Therefore, it is necessary to estimate the stability of reference genes at various altitudes.

In the present study, eight different reference genes were selected to be assessed and validated for stability at different altitudes using the geNorm, NormFinder, BestKeeper and RefFinder programs. The study identified two candidate genes (PPIA and RPL13A) that are stably 
205

206

207

208

209

210

211

212

213

214

215

216

217

218

219

220

221

222

223

224

225

226

227

228

229

230

231

232

233

234

235

236

237

238

239

240

241

242

243

244

245

expressed under hypoxic stress and can be used as reference genes for relative gene quantification and normalization before and after entering the plateau region.

In this study, three widely used algorithms (geNorm, NormFinder and BestKeeper) were applied to calculate the stability of the selected reference gene expression levels. The geNorm algorithm uses the principle that the expression ratio of two ideal reference genes is identical in all tested samples (Vandesompele et al. 2002). According to the average pairwise variation of one reference gene with all other candidate genes, a lower M-value indicates greater stability of the candidate gene. NormFinder, which is based on the stability value of the internal control genes, can select the minimally fluctuating genes as the most stable genes, but it can only select one suitable reference gene for normalization. The ranking results varied across the different algorithms. The comprehensive RefFinder ranking indicated that GAPDH and PPIA were the most stable genes in the plains and plateau groups, respectively, and PPIA was the most stable gene in both stages.

Previous studies have reported that $\beta 2-M G$ levels do not vary with oxygen concentration (Petousi et al. 2014). Studies in bladder cancer cells under hypoxia showed that $\beta 2-M G$ and Hypoxanthine phosphoribosyltransferase-1 (HPRT) were the most suitable reference genes for normalizing gene expression (Lima et al. 2016). In human retinal endothelial cells, TBP and pumilio RNA binding family member 1 (PUMI) were the most stable reference genes under hypoxic conditions (Xie et al. 2016). However, the present study showed that the stress-specific candidate genes $\beta 2$ $M G$ and $T B P$ were not suitable for normalizing target gene expression in blood under normoxic and hypoxic conditions.

Under normoxic conditions, GAPDH was the most stable gene in the blood, whereas under hypoxic conditions, PPIA was the most stable candidate reference gene. RPL13A was ranked as the second most stable reference gene in blood both under normoxic and hypoxic conditions. $A C T B$ was observed to be the most stable candidate gene in plain blood using the geNorm algorithm (Fig. 3B), but it was the least stable (Fig. 3A) in the combined analysis of tested samples. In the plateau stage but not in the plains stage, 18S RNA was one of the most stable genes. The differences in the reference gene rankings could be associated with the algorithms used by each program.

Our study has some limitations. The identification of stable candidate genes for target gene expression analysis in human blood between low- and high-altitude conditions was a major challenge due to the difficulty involved in sample collection. This difficulty may account for the limited number of volunteers enrolled in the present study and the limited number of gene expression studies of blood in the plateau environment. Thus, one of the limitations of this study was that we could not collect enough blood samples to strengthen the reliability of the present study. In addition, analyses of the stability of reference gene expression should be verified at a cellular level in a hypoxic chamber. In the present study, however, the stability of candidate reference genes was reliably evaluated in blood under normoxic and hypoxic stress conditions using algorithms. Previous studies on target gene expression analyses of blood under hypoxic conditions used $18 S R N A$ (Mishra et al. 2013) and $\beta 2-M G$ (Petousi et al. 2014) as reference genes for normalization. The present study clearly showed that both PPIA and RPL13A are stable 
246

247

248

249

250

251

252

253

254

255

256

257

258

259

260

261

262

263

264

265

266

267

and suitable reference genes, but the amplification efficiency of PPIA was more than 1.05 (Table 2). Thus, RPL13A is the most suitable and stable reference gene for the normalization of target gene expression in blood from the plains and plateau environments.

In conclusion, the present study determined that GAPDH and RPL13A in blood from the plains region and PPIA and RPL13A in blood from the plateau region were the most stable reference genes. Among the identified stably expressed reference genes in both the plains and plateau environments, RPL13A was shown to be most stable in blood from both the normoxic and hypoxic conditions. Additional studies should be conducted on the cellular level to verify the stability of the same reference genes.

\section{Conclusions}

In this study, the expression levels of eight candidate human reference genes (GAPDH, ACTB, $18 S$ RNA, $\beta 2-M G, P P I A, R P L 13 A, T B P$ and SDHA) were assessed in blood from hypoxic environments. We determined, for the first time, that $R P L 13 A$ was the most reliable reference gene for the normalization of target gene expression in human blood from low- and high-altitude environments. However, to obtain reliable data, the use of more than one reference gene is strongly recommended.

\section{Acknowledgements}

The authors thank Fengyan Fan for excellent technical assistance and we also thank the members of the Department of Gene Detection in our hospital for helpful discussion.

\section{Availability of data and materials}

All data supporting the conclusion of this article are contained within the manuscript.

\section{Reference}

Ahn K, Huh JW, Park SJ, Kim DS, Ha HS, Kim YJ, Lee JR, Chang KT, and Kim HS. 2008. Selection of internal reference genes for SYBR green qRT-PCR studies of rhesus monkey (Macaca mulatta) tissues. BMC Mol Biol 9:78. 10.1186/1471-2199-9-78

Andersen CL, Jensen JL, and Orntoft TF. 2004. Normalization of real-time quantitative reverse transcriptionPCR data: a model-based variance estimation approach to identify genes suited for normalization, applied to bladder and colon cancer data sets. Cancer Res 64:5245-5250. 10.1158/0008-5472.CAN04-0496

Bakhashab S, Lary S, Ahmed F, Schulten HJ, Bashir A, Ahmed FW, Al-Malki AL, Jamal HS, Gari MA, and Weaver JU. 2014. Reference genes for expression studies in hypoxia and hyperglycemia models in human umbilical vein endothelial cells. G3 (Bethesda) 4:2159-2165. 10.1534/g3.114.013102

Beall CM. 2000. Tibetan and Andean patterns of adaptation to high-altitude hypoxia. Hum Biol 72:201-228.

Beall CM, Cavalleri GL, Deng L, Elston RC, Gao Y, Knight J, Li C, Li JC, Liang Y, McCormack M, Montgomery HE, Pan H, Robbins PA, Shianna KV, Tam SC, Tsering N, Veeramah KR, Wang W, 
Wangdui P, Weale ME, Xu Y, Xu Z, Yang L, Zaman MJ, Zeng C, Zhang L, Zhang X, Zhaxi P, and Zheng YT. 2010. Natural selection on EPAS1 (HIF2alpha) associated with low hemoglobin concentration in Tibetan highlanders. Proc Natl Acad Sci U S A 107:11459-11464. 10.1073/pnas. 1002443107

Bustin SA. 2002. Quantification of mRNA using real-time reverse transcription PCR (RT-PCR): trends and problems. J Mol Endocrinol 29:23-39.

Bustin SA, Benes V, Garson JA, Hellemans J, Huggett J, Kubista M, Mueller R, Nolan T, Pfaffl MW, Shipley GL, Vandesompele J, and Wittwer CT. 2009. The MIQE guidelines: minimum information for publication of quantitative real-time PCR experiments. Clin Chem 55:611-622. 10.1373/clinchem.2008.112797

Bustin SA, and Nolan T. 2004. Pitfalls of quantitative real-time reverse-transcription polymerase chain reaction. J Biomol Tech 15:155-166.

Chen G, Yue A, Yu H, Ruan Z, Yin Y, Wang R, Ren Y, and Zhu L. 2016. Mesenchymal Stem Cells and Mononuclear Cells From Cord Blood: Cotransplantation Provides a Better Effect in Treating Myocardial Infarction. Stem Cells Transl Med 5:350-357. 10.5966/sctm.2015-0199

Fjeldbo CS, Aarnes EK, Malinen E, Kristensen GB, and Lyng H. 2016. Identification and Validation of Reference Genes for RT-qPCR Studies of Hypoxia in Squamous Cervical Cancer Patients. PLoS One 11:e0156259. 10.1371/journal.pone.0156259

Gentile AM, Lhamyani S, Coin-Araguez L, Oliva-Olivera W, Zayed H, Vega-Rioja A, Monteseirin J, RomeroZerbo SY, Tinahones FJ, Bermudez-Silva FJ, and El Bekay R. 2016. RPL13A and EEF1A1 Are Suitable Reference Genes for qPCR during Adipocyte Differentiation of Vascular Stromal Cells from Patients with Different BMI and HOMA-IR. PLoS One 11:e0157002. 10.1371/journal.pone.0157002

Jaramillo ML, Ammar D, Quispe RL, Guzman F, Margis R, Nazari EM, and Muller YM. 2017. Identification and evaluation of reference genes for expression studies by RT-qPCR during embryonic development of the emerging model organism, Macrobrachium olfersii. Gene 598:97-106. 10.1016/j.gene.2016.11.001

Ji LD, Qiu YQ, Xu J, Irwin DM, Tam SC, Tang NL, and Zhang YP. 2012. Genetic adaptation of the hypoxiainducible factor pathway to oxygen pressure among eurasian human populations. Mol Biol Evol 29:3359-3370. 10.1093/molbev/mss144

Lima L, Gaiteiro C, Peixoto A, Soares J, Neves M, Santos LL, and Ferreira JA. 2016. Reference Genes for Addressing Gene Expression of Bladder Cancer Cell Models under Hypoxia: A Step Towards Transcriptomic Studies. PLoS One 11:e0166120. 10.1371/journal.pone.0166120

Liu LL, Zhao H, Ma TF, Ge F, Chen CS, and Zhang YP. 2015. Identification of valid reference genes for the normalization of RT-qPCR expression studies in human breast cancer cell lines treated with and without transient transfection. PLoS One 10:e0117058. 10.1371/journal.pone.0117058

Liu X, Xie J, Liu Z, Gong Q, Tian R, and Su G. 2016. Identification and validation of reference genes for quantitative RT-PCR analysis of retinal pigment epithelium cells under hypoxia and/or hyperglycemia. Gene 580:41-46. 10.1016/j.gene.2016.01.001

Mishra A, Mohammad G, Thinlas T, and Pasha MA. 2013. EGLN1 variants influence expression and $\mathrm{SaO} 2$ levels to associate with high-altitude pulmonary oedema and adaptation. Clin Sci (Lond) 124:479-489. $10.1042 / \mathrm{CS} 20120371$ 
Nolan T, Hands RE, and Bustin SA. 2006. Quantification of mRNA using real-time RT-PCR. Nat Protoc 1:1559-1582. 10.1038/nprot.2006.236

Ofinran O, Bose U, Hay D, Abdul S, Tufatelli C, and Khan R. 2016. Selection of suitable reference genes for gene expression studies in normal human ovarian tissues, borderline ovarian tumours and ovarian cancer. Mol Med Rep 14:5725-5731. 10.3892/mmr.2016.5933

Petousi N, Croft QP, Cavalleri GL, Cheng HY, Formenti F, Ishida K, Lunn D, McCormack M, Shianna KV, Talbot NP, Ratcliffe PJ, and Robbins PA. 2014. Tibetans living at sea level have a hyporesponsive hypoxia-inducible factor system and blunted physiological responses to hypoxia. J Appl Physiol (1985) 116:893-904. 10.1152/japplphysiol.00535.2013

Pfaffl MW, Tichopad A, Prgomet C, and Neuvians TP. 2004. Determination of stable housekeeping genes, differentially regulated target genes and sample integrity: BestKeeper--Excel-based tool using pairwise correlations. Biotechnol Lett 26:509-515.

Polotsky VY, Bevans-Fonti S, Grigoryev DN, and Punjabi NM. 2015. Intermittent Hypoxia Alters Gene Expression in Peripheral Blood Mononuclear Cells of Healthy Volunteers. PLoS One 10:e0144725. 10.1371/journal.pone.0144725

Sellars MJ, Vuocolo T, Leeton LA, Coman GJ, Degnan BM, and Preston NP. 2007. Real-time RT-PCR quantification of Kuruma shrimp transcripts: a comparison of relative and absolute quantification procedures. J Biotechnol 129:391-399. 10.1016/j.jbiotec.2007.01.029

Srikanth L, Sunitha MM, Venkatesh K, Kumar PS, Chandrasekhar C, Vengamma B, and Sarma PV. 2015. Anaerobic Glycolysis and HIF1alpha Expression in Haematopoietic Stem Cells Explains Its Quiescence Nature. J Stem Cells 10:97-106.

Tricarico C, Pinzani P, Bianchi S, Paglierani M, Distante V, Pazzagli M, Bustin SA, and Orlando C. 2002. Quantitative real-time reverse transcription polymerase chain reaction: normalization to rRNA or single housekeeping genes is inappropriate for human tissue biopsies. Anal Biochem 309:293-300.

Valasek MA, and Repa JJ. 2005. The power of real-time PCR. Adv Physiol Educ 29:151-159. 10.1152/advan.00019.2005

Vandesompele J, De Preter K, Pattyn F, Poppe B, Van Roy N, De Paepe A, and Speleman F. 2002. Accurate normalization of real-time quantitative RT-PCR data by geometric averaging of multiple internal control genes. Genome Biol 3:RESEARCH0034.

Wang H, Yang B, Geng T, Li B, Dai P, and Chen C. 2015. Tissue-specific selection of optimal reference genes for expression analysis of anti-cancer drug-related genes in tumor samples using quantitative real-time RT-PCR. Exp Mol Pathol 98:375-381. 10.1016/j.yexmp.2014.10.014

Xie J, Liu X, Li Y, Liu Y, and Su G. 2016. Validation of RT-qPCR reference genes and determination of Robo4 expression levels in human retinal endothelial cells under hypoxia and/or hyperglycemia. Gene 585:135-142. 10.1016/j.gene.2016.03.047

Zhang X, Ding L, and Sandford AJ. 2005. Selection of reference genes for gene expression studies in human neutrophils by real-time PCR. BMC Mol Biol 6:4. 10.1186/1471-2199-6-4 
Figure 1

\section{Specificity of RT-qPCR amplicons}

Dissociation curves with single peaks were generated from all amplicons and showed no dimer formation for each reference gene.
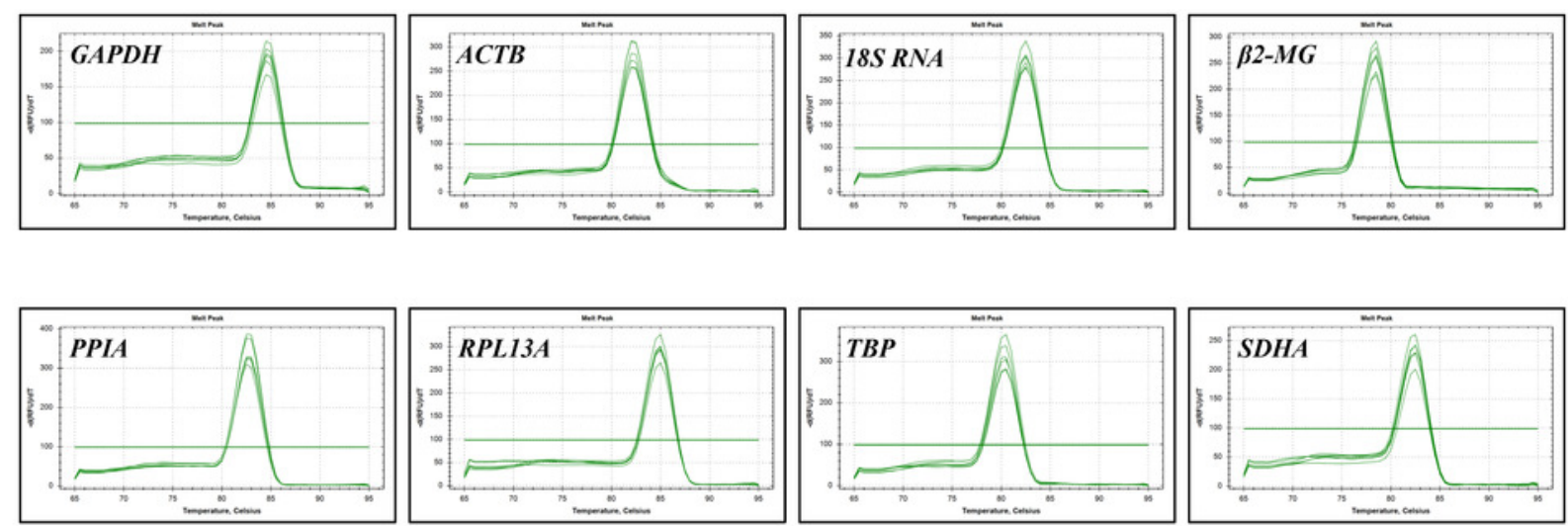
Figure 2

Candidate reference gene $\mathrm{Ct}$ value distributions.

Boxplots of the Ct values from six volunteers from the plain (A) and the plateau (B) stages for each of the eight candidate reference genes.
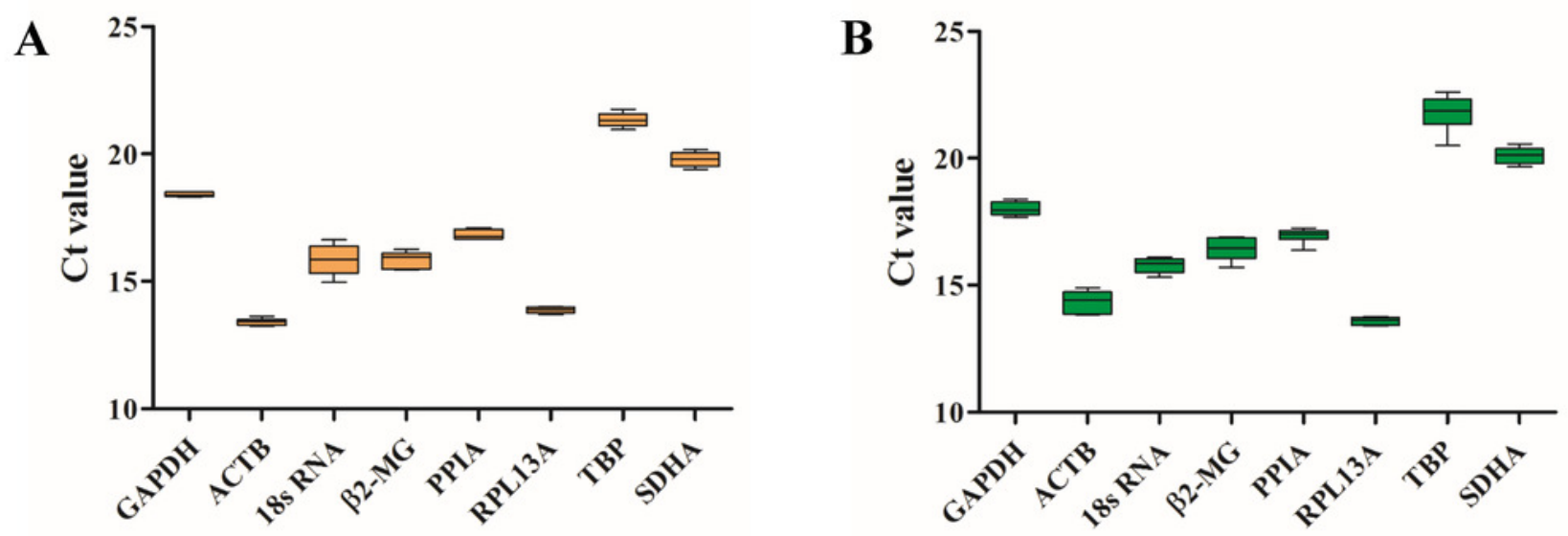
Figure 3

The geNorm selection analysis of candidate reference genes.

The average expression stability value (M) was calculated by geNorm for each gene on the plain (A), plateau (B) or both stages (C). Pairwise variation (V) between the normalization factors ( $V n$ and $V n+1)$ was used to determine the optimal number of reference genes for normalization on the plain (D), plateau $(E)$ or both stages $(F)$.
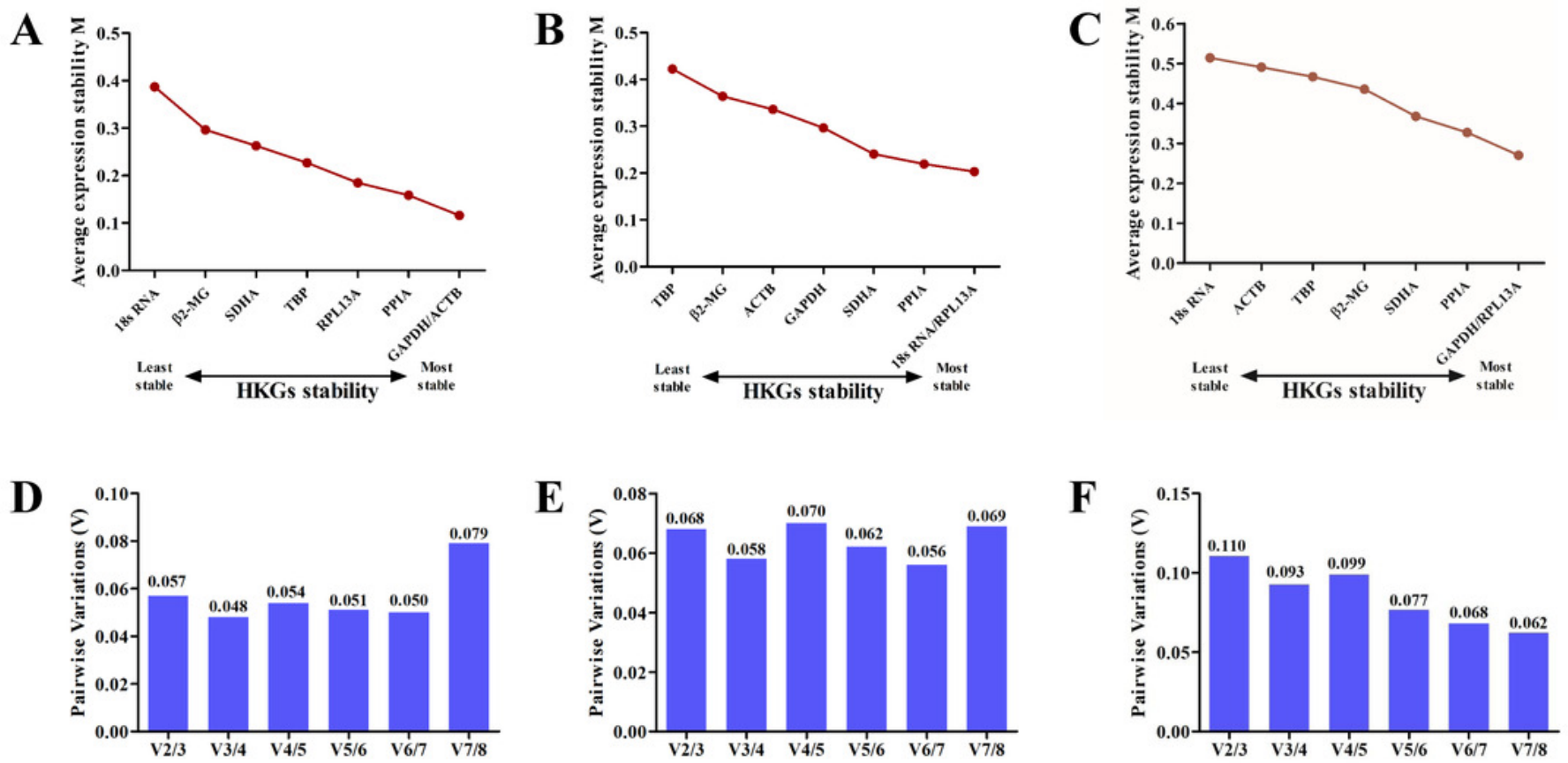


\section{Table 1 (on next page)}

Primer sequence information for RT-qPCR amplification used in this study 
Table1:Primer sequence information for RT-qPCR amplification used in this study

\begin{tabular}{|c|c|c|c|c|c|c|c|}
\hline Symbol & Gene Name & $\begin{array}{l}\text { Accession } \\
\text { Number }\end{array}$ & Forward Primer Sequence [5'-3'] & $\begin{array}{c}\text { Position in } \\
\text { cDNA }\end{array}$ & Reverse Primer Sequence [5'-3'] & $\begin{array}{c}\text { Position in } \\
\text { cDNA }\end{array}$ & $\begin{array}{c}\text { Production } \\
\text { Size }\end{array}$ \\
\hline GAPDH & Glyceraldehyde & NM_002046.5 & TCCAAAATCAAGTGGGGCGA & 4th exon & TGATGACCCTTTTGGCTCCC & 5th exon & $115 \mathrm{bp}$ \\
\hline ACTB & $\beta$-actin & NM_001101.3 & CTTCCAGCCTTCCTTCCTGG & 4th exon & CTGTGTTGGCGTACAGGTCT & 5 th exon & $110 \mathrm{bp}$ \\
\hline 18s RNA & 18s RNA & M_10098.1 & GGAGCCTGCGGCTTAATTTG & & CCACCCACGGAATCGAGAAA & & $100 \mathrm{bp}$ \\
\hline$\beta 2-\mathrm{MG}$ & $\beta 2$-microglobulin & NM_004048.2 & TGGGTTTCATCCATCCGACA & 2 th exon & TCAGTGGGGGTGAATTCAGTG & 2 exon & $138 b p$ \\
\hline PPIA & Peptidylprolylisomerase A & NM_021130.3 & GACTGAGTGGTTGGATGGCA & 4th exon & TCGAGTTGTCCACAGTCAGC & 5 th exon & $141 b p$ \\
\hline RPL13A & Ribosomal protein L13 & NM_012423.3 & AAAAGCGGATGGTGGTTCCT & 6th exon & GCTGTCACTGCCTGGTACTT & 7th exon & $118 b p$ \\
\hline TBP & TATA-Box binding protein & NM_003194.4 & CAGCTTCGGAGAGTTCTGGG & 3th exon & TATATTCGGCGTTTCGGGCA & 4 th exon & $117 \mathrm{bp}$ \\
\hline SDHA & $\begin{array}{l}\text { Succinate dehydrogenase } \\
\text { complex, subunit A }\end{array}$ & NM_004168.3 & AAACTCGCTCTTGGACCTGG & 10th exon & TCTTCCCCAGCGTTTGGTTT & 11th exon & $111 \mathrm{bp}$ \\
\hline
\end{tabular}


Table 2 (on next page)

RT-qPCR analysis for determination of the amplification efficiency

E:efficiency $R^{2}$ :correlation coefficient body 
1 Table2: RT-qPCR analysis for determination of the amplification efficiency

\begin{tabular}{lccc}
\hline Gene & Slope & $\mathrm{E}(\%)$ & $\mathrm{R}^{2}$ \\
\hline GAPDH & -3.162 & 107.1 & 0.999 \\
ACTB & -3.432 & 95.6 & 0.997 \\
18s RNA & -3.422 & 96.0 & 0.998 \\
B2-MG & -3.302 & 100.8 & 0.998 \\
PPIA & -3.014 & 114.7 & 0.990 \\
RPL13A & -3.254 & 102.9 & 0.999 \\
TBP & -3.227 & 104.1 & 0.997 \\
SDHA & -3.199 & 105.4 & 0.997 \\
\hline E:efficiency & \multicolumn{3}{l}{$\mathrm{R}^{2}$ :correlation coefficient }
\end{tabular}




\section{Table 3(on next page)}

Descriptive statistics and normality evaluation of the reference genes $\mathrm{Cq}$ values before and after entering plateau

$\mathrm{SD}$, standard deviation; Min Cq, minimum Cq value; Max Cq, maximum Cq value; SW-test $p$, p-value of the Shapiro-Wilk test. 
1 Table3 Descriptive statistics and normality evaluation of the reference genes Cq values before and

2 after entering plateau

\begin{tabular}{rlllcccc}
3 & & Gene & Mean & SD & Min Cq & Max Cq & SW-test $p$ \\
\cline { 2 - 7 } 5 & Before & GAPDH & 18.41 & 0.09 & 18.31 & 18.52 & 0.248 \\
6 & plateau & ACTB & 13.40 & 0.13 & 13.25 & 13.63 & 0.601 \\
7 & & 18s RNA & 15.84 & 0.56 & 14.79 & 16.63 & 0.989 \\
8 & & B2-MG & 15.86 & 0.30 & 15.46 & 16.25 & 0.326 \\
9 & & PPIA & 16.82 & 0.18 & 16.66 & 17.09 & 0.095 \\
10 & & RPL13A & 13.88 & 0.10 & 13.71 & 14.00 & 0.620 \\
11 & & TBP & 21.34 & 0.26 & 20.96 & 21.75 & 0.996 \\
12 & & SDHA & 19.79 & 0.26 & 19.41 & 20.17 & 0.963 \\
13 & After & GAPDH & 18.01 & 0.28 & 17.68 & 18.40 & 0.664 \\
14 & plateau & ACTB & 14.35 & 0.43 & 13.84 & 14.89 & 0.526 \\
15 & & 18s RNA & 15.79 & 0.29 & 15.32 & 16.10 & 0.616 \\
16 & & B2-MG & 16.43 & 0.45 & 15.71 & 16.90 & 0.661 \\
17 & & PPIA & 16.96 & 0.30 & 16.38 & 17.26 & 0.089 \\
18 & & RPL13A & 13.60 & 0.15 & 13.39 & 13.77 & 0.486 \\
19 & & TBP & 21.78 & 0.73 & 20.50 & 22.60 & 0.530 \\
20 & & SDHA & 20.11 & 0.32 & 19.67 & 20.57 & 0.987 \\
\hline
\end{tabular}

22 SD, standard deviation; Min Cq, minimum Cq value; Max Cq, maximum Cq value; SW-test p, p23 value of the Shapiro-Wilk test. 
Table 4(on next page)

Calculation of candidate reference genes expression stability by the NormFinder. 
1 Table4: Calculation of candidate reference genes expression stability by the NormFinder

\begin{tabular}{clclccc}
\hline $\begin{array}{c}\text { Ranking } \\
\text { order }\end{array}$ & Gene & $\begin{array}{c}\text { Stability value } \\
\text { (Whole stages) }\end{array}$ & Gene & $\begin{array}{c}\text { Stability value } \\
\text { (Plain) }\end{array}$ & Gene & $\begin{array}{c}\text { Stability value } \\
\text { (plateau) }\end{array}$ \\
\hline 1 & PPIA & 0.080 & GAPDH & 0.032 & PPIA & 0.076 \\
2 & SDHA & 0.136 & RPL13A & 0.057 & SDHA & 0.166 \\
3 & TBP & 0.205 & ACTB & 0.110 & ACTB & 0.176 \\
4 & RPL13A & 0.227 & PPIA & 0.121 & RPL13A & 0.182 \\
5 & 18s RNA & 0.229 & TBP & 0.167 & 18 RNA & 0.194 \\
6 & B2-MG & 0.237 & SDHA & 0.210 & GAPDH & 0.237 \\
7 & GAPDH & 0.265 & B2-MG & 0.264 & $\beta 2-$ MG & 0.244 \\
8 & ACTB & 0.316 & 18s RNA & 0.434 & TBP & 0.369 \\
\hline
\end{tabular}

2 


\section{Table 5 (on next page)}

Results from BestKeeper analysis. 
1 Table5: Results from BestKeeper analysis

\begin{tabular}{|c|c|c|c|c|}
\hline Gene & & Whole Stage & Plain & Plateau \\
\hline \multirow[t]{2}{*}{ GAPDH } & $\operatorname{std} \operatorname{dev}[ \pm \mathrm{CP}]$ & 0.24 & 0.08 & 0.23 \\
\hline & $\mathrm{CV}[\% \mathrm{CP}]$ & 1.33 & 0.41 & 1.25 \\
\hline \multirow[t]{2}{*}{ ACTB } & std dev $[ \pm \mathrm{CP}]$ & 0.48 & 0.11 & 0.35 \\
\hline & $\mathrm{CV}[\% \mathrm{CP}]$ & 3.45 & 0.82 & 2.45 \\
\hline \multirow[t]{2}{*}{ 18s RNA } & std dev $[ \pm \mathrm{CP}]$ & 0.33 & 0.44 & 0.23 \\
\hline & $\mathrm{CV}[\% \mathrm{CP}]$ & 2.09 & 2.79 & 1.45 \\
\hline \multirow[t]{2}{*}{$\beta 2-\mathrm{MG}$} & $\operatorname{std} \operatorname{dev}[ \pm \mathrm{CP}]$ & 0.37 & 0.26 & 0.33 \\
\hline & $\mathrm{CV}[\% \mathrm{CP}]$ & 2.31 & 1.63 & 2.04 \\
\hline \multirow[t]{2}{*}{ PPIA } & $\operatorname{std} \operatorname{dev}[ \pm \mathrm{CP}]$ & 0.21 & 0.16 & 0.19 \\
\hline & $\mathrm{CV}[\% \mathrm{CP}]$ & 1.24 & 0.93 & 1.15 \\
\hline \multirow[t]{2}{*}{ RPL13A } & $\operatorname{std} \operatorname{dev}[ \pm \mathrm{CP}]$ & 0.15 & 0.09 & 0.12 \\
\hline & $\mathrm{CV}[\% \mathrm{CP}]$ & 1.10 & 0.62 & 0.89 \\
\hline \multirow[t]{2}{*}{ TBP } & std dev $[ \pm \mathrm{CP}]$ & 0.43 & 0.22 & 0.53 \\
\hline & $\mathrm{CV}[\% \mathrm{CP}]$ & 1.98 & 1.01 & 2.42 \\
\hline \multirow[t]{2}{*}{ SDHA } & std dev $[ \pm \mathrm{CP}]$ & 0.27 & 0.23 & 0.25 \\
\hline & $\mathrm{CV}[\% \mathrm{CP}]$ & 1.34 & 1.15 & 1.22 \\
\hline
\end{tabular}

2 
Table 6(on next page)

Stabilities of HKGs ranked by RefFinder. 
1 Table6: Stabilities of HKGs ranked by RefFinder

2

\begin{tabular}{ccll}
\hline Ranking Order & Whole Stages & Plain & Plateau \\
\hline 1 & PPIA & GAPDH & PPIA \\
2 & RPL13A & RPL13A & RPL13A \\
3 & SDHA & ACTB & 18 sRNA \\
4 & GAPDH & PPIA & SDHA \\
5 & $\beta 2-M G$ & TBP & GAPDH \\
6 & TBP & SDHA & ACTB \\
7 & 18 sRNA & $\beta 2-M G$ & $\beta 2-M G$ \\
8 & ACTB & 18 sRNA & TBP \\
\hline
\end{tabular}

